\title{
A new in vivo model for analysis of colon carcinoma micrometastasis in BALB/c mice
}

\author{
Roya Abedizadeh $^{\mathbb{1}}$, Hossein Rezvan ${ }^{1 *}{ }^{\mathbb{D}}$, Alireza Nourian ${ }^{1}$ \\ ${ }^{1}$ Department of Pathobiology, Faculty of Paraveterinary Sciences, Bu-Ali Sina University, Hamadan, Iran.
}

*Corresponding Author: Hossein Rezvan, Department of Pathobiology, Faculty of Paraveterinary Sciences, Bu-Ali Sina University, Hamadan, Iran ;Tel: 09187103745, Fax: 08134227350, Email: h.rezvan@basu.ac.ir

\begin{abstract}
Background and aims: Colorectal cancer (CRC) is known as the fourth leading cause of death across the world. The fate of patients depends on the metastatic spread of cancer cells. Micrometastases are small clusters of cancer cells with no diagnostic evidence during diagnosis and surgery. Therefore, experimental models for micrometastasis are necessary to investigate tumors. We developed a mouse model to evaluate micrometastasis of colon carcinoma cells by systemic injection of tumor cells.

Methods: In this study, stably transfected CT26 cells expressing Leishmania major GP63 were intravenously (IV) injected into BALB/c mice for induction of micrometastases. The mice were divided into three groups and the groups were sacrificed on days 3,7 , and 14 of the injection. reverse transcriptase-polymerase chain reaction (RT-PCR) was performed on tissue samples to detect Gp63 gene.

Results: Our results showed the successful construction and transfection of pcDNA3 L. major Gp63 into CT26 cells. After IV injection, total cellular RNA was extracted and the Gp63 gene was detected in the liver, lung, and kidney but not in the colon.

Conclusion: Due to the significance of micrometastasis and the need to establish simple models for cancer research, an experimental mouse model was developed. CT26 tumor cells stably expressing Gp63 generated a potent system for diagnosis of micrometastatic cells in tissues. Injection into the tail vein is a practical model for cancer research because of the lower fatality rate and no need for anesthesia. Keywords: Colon carcinoma, (Micro) Metastasis, Mouse model, BALB/C
\end{abstract}

Received: 24 September 2019, Accepted: 28 December 2019, ePublished: 29 April 2020

\section{Introduction}

Colorectal cancer (CRC) is known as the third leading neoplasm in both men and women worldwide (1) which has a $12 \%$ five-year survival rate when it becomes metastatic (2). CRC accounts for over $9.7 \%$ of all cancer cases (3). Reportedly, it has equal prevalence among men and women with over 1.2 million new cases in 2008 (4), which increased to 1.8 million in 2018 (5). In Iran, $\mathrm{CRC}$ is the third leading cancer in men and the fourth in women (6). It is the third leading cause of death due to cancer among non-smokers (7).

The majority of tumors derived from epithelial tissues are solid such as breast, lung, and gastrointestinal tract carcinomas (8). The fate of the patients with these tumors is largely dependent on the metastatic propagation of tumor cells (9). Micrometastases are small clusters of cancer cells ( 4 to 16 cells) (10) with no diagnostic evidence during diagnosis and surgery (11). Micrometastases that do not respond to common therapies will usually lead to cancer relapses (12). Therefore, experimental models of micrometastasis are necessary for diagnostic and therapeutic investigation of tumor. In most mouse models, the tumor cells are injected intraportally to target the liver (10). The infusion of cancer cells within the left ventricle of mice heart also imitates the process of micrometastasis (12). Subcutaneous xenografts may lead to the formation of experimental metastases (13). Different methods can be used to detect micrometastasis in these models including in vivo video microscopy, bioluminescent reporter imaging (12), fluorescence imaging using tumor cells expressing green fluorescent protein (14), molecular detection using reverse transcriptase-polymerase chain reaction (RT-PCR) (15) and immunohistochemical markers for proliferation and apoptosis in tumor cells (10).

In this study, we introduced a model to assess experimental hepatic and pulmonary (micro) metastasis of CT26 colon carcinoma cells in syngeneic BALB/c mice by systemic injection of CT26 tumor cells expressing Leishmania glycoprotein 63 (Gp63) gene into the tail vein. This model mimics the movement of tumor cells from a primitive tumor region to other remote sites through blood circulation. It has been shown that tumor metastases develop in specific locations. Therefore, the homing of circulating tumor cells in other organs cannot randomly 
occur (16)

To label and track the CT26 tumor cells in mice tissues, the cells were transfected with Leishmania major Gp63 which is a preserved gene only in Leishmania parasites (17). The Gp63 gene was verified by DNA sequencing and cloned into the pcDNA3 mammalian expression vector and then transfected into CT26 cells using Lipofectamine 2000, a cationic liposome which allows the nucleic acid molecules to enter the cells (18). Gp63 gene exists neither in tumor cells nor in the mouse genome; therefore, it can serve as a reliable marker to detect tumor cells in different tissues.

Advances in molecular oncology have provided sensitive, specific and cost-effective methods to diagnose metastatic diseases. RT-PCR is thought to be capable of detecting micrometastases (19) as it is able to detect 1 cancer cell in $10^{6}$ normal cells (20). Therefore, given the advantages of this technique, RT-PCR was used to detect CT26 L. major Gp63+ cells.

Our study represents an allograft mouse model of micrometastatic colon carcinoma that leaves the immune system intact and acts as a normal body system. This technique is practicable and does not need anesthesia and surgery.

\section{Materials and Methods}

Cells

In this experimental study, CT26, an epithelial colon carcinoma cell line, of BALB/c mice (21) was provided by Pasteur Institute of Iran, Tehran, Iran. The cells were cultured by RPMI 1640 (Bio Idea, Tehran, Iran) with $10 \%$ fetal bovine serum (FBS) (Bio Idea, Tehran, Iran) in addition of $1 \%$ penicillin/streptomycin (Bio Idea, Tehran, Iran) and incubated at $37^{\circ} \mathrm{C}$ with $5 \% \mathrm{CO}_{2}(22)$.

\section{Animals}

6-8 week old male BALB/c mice were prepared from Pasteur Institute, Tehran, Iran and housed in animal house of Bu-Ali Sina University with free access to fresh water and food. Three mice were used to induce subcutaneous tumors and twelve mice for intravenous injection.

\section{Antibiotic sensitivity assay}

A serial concentration of G418 (neomycin) (SigmaAldrich, St. Louis, USA) from 500 to $2500 \mu \mathrm{g} / \mathrm{mL}$ was added to 24-well plates (JET BIOFIL, Seoul, South Korea) containing $10^{6}$ CT26 cells/well; each concentration was run in duplicate followed by a ten day incubation at $37^{\circ} \mathrm{C}$ with 5\% CO2. The lowest concentration of G418 (1500 $\mu \mathrm{g} / \mathrm{mL}$ ) in which all the tumor cells died within 7-10 days was selected for transfection.

Transfection of CT26 tumor cells with L. major Gp63 CT26 tumor cells were transfected with pcDNA3 vector containing L. major Gp63 by Lipofectamine 2000
(Invitrogen, Carlsbad, USA). Briefly, pcDNA3 L. major Gp63 with Lipofectamine 2000 were mixed in RPMI1640 media in concentrations of $1 \mu \mathrm{g} / 50 \mu \mathrm{L}$ and $3 \mu \mathrm{g} / 50 \mu \mathrm{L}$, respectively. The DNA with Lipofectamine solutions were mixed together properly followed by incubation at room temperature for 5 minutes and added to the CT26 cells at concentration of $10^{6}$ cells/well in 24 -well plates after removing the cell culture supernatant. The cells were incubated in $\mathrm{CO}_{2}$ incubator for 30 minutes. Then, $1 \mathrm{~mL}$ of RPMI 1640 supplemented with 10\% FBS was added to the cells and after 24 hours, the media was replaced with a new media supplemented with 10\% FBS and $1500 \mu \mathrm{g} /$ mL G418.

\section{Establishment of stably transfected CT26 cell line}

In order to generate single cell clones, transfected CT26 cells were harvested and cultured in a 96-well plate (JET BIOFIL, Seoul, South Korea) at a concentration of one cell per well in $100 \mu \mathrm{L}$ of RPMI 1640 medium with $10 \%$ FBS, $1 \%$ penicillin/streptomycin, and $1500 \mu \mathrm{g} / \mathrm{mL}$ G418. The cells were incubated in a cell culture incubator, and after 3 weeks, clones with the highest growth rate were identified and cultured in complete media with G418. The cells were finally checked for L. major Gp63 gene expression by RTPCR.

\section{Induction of subcutaneous tumors and histopathology analysis}

Confluent cultures of stably transfected CT26 L. major Gp63+ cells were harvested with trypsinization, washed several times with sterile phosphate-buffered saline (PBS) buffer and resuspended at a final concentration of $10^{6}$ cells/100 $\mu \mathrm{L}$ PBS. The cells were infused into the right flank of animals subcutaneously and observed daily for tumor growth. The subcutaneous tumors were measured using a ruler.

After 4 weeks, the animals were euthanized. Then, a small piece of skin at the injection site from each animal was cut and fixed with formalin. The tissues were then dehydrated, cleared, paraffin-embedded, sectioned at $5 \mu \mathrm{m}$ thickness, placed on a glass slide and stained with hematoxylin and eosin. The sections were examined independently and photographed by a veterinary pathologist using a light microscope (Olympus CX41, Tokyo, Japan) equipped with a digital camera (Olympus DP25, Hamburg, Germany).

\section{Induction of micrometastases by intravenous injection of cancer cells}

Twelve male BALB/c mice were included in this study. One group of nine mice were injected with $10^{6}$ cells $/ 100$ $\mu \mathrm{L}$ of stably transfected CT26 L. major $G p 63^{+}$as test group and one group of three was injected only with PBS as control group. The cells were injected into the tail vein of the animals (23). On days 3, 7, and 14 after injection, three mice of test group as well as one mouse of the control 
group were sacrificed in each day $(10,24)$. The livers, lungs, kidneys, and colons were excised and examined for the presence of CT26 micrometastasis by detecting $L$. major Gp63 RNA.

\section{Cloning of L. major complementary DNA (cDNA) coding for Gp63}

Cytomegalovirus promoter pNUT L. major Gp63 DNA was kindly received from Professor Mack Master (Department of Medical Genetics, University of British Columbia, Vancouver, Canada). First, pNUT L. major Gp63 DNA was amplified by Escherichia coli transformation and the Gp63 clone was verified by sequencing and aligning the sequence of the DNA with that accessible in the gene bank (accession number Y00647).

5'- TCTAAGCTTCACCCGATCCATGTGCCG-3' containing HindIII as forward and 5'AGAATTCCTGCACACTGGCGGCCGTTA -3' containing EcoRI as reverse primers were used to amplify the cDNA fragment by PCR. The PCR product of $L$. major Gp63 gene holding the restriction sites on both sides and pcDNA3 vector (Invitrogen, Carlsbad, USA) were digested with appropriate restriction enzymes (RNA Biotechnology Company, Esfahan, Iran), and then loaded on agarose gel. After cutting the gene and the vector, they were extracted from the gel using DNA extraction kit (RNA Biotechnology Company, Esfahan, Iran) according to the manufacturer's instructions. Then, the extracted molecules were ligated using a ligation enzyme (Promega, Durham, USA). The pcDNA3 L. major Gp63 vector was multiplied and extracted by E. coli transformation (25).

\section{Extraction and Detection of L. major Gp63 RNA}

For tracing L. major Gp63 RNA in transfected cells and also mouse tissue samples from livers, lungs, kidneys, and colons, total RNA was extracted from the cells by Iraizol kit (RNA Biotechnology Company, Esfahan, Iran) according to the producer's instructions. Briefly, CT26 cells were cultured in T25 cell culture flasks and harvested by trypsinization and centrifuged at $420 \mathrm{G}$ (trypsin 0.1\% (Bio Idea, Tehran, Iran) and EDTA 0.02\% (Merck, Germany). For the extraction of tissue RNA, the samples were first chopped up and then squashed. At the next step, $1 \mathrm{~mL}$ of Iraizol (RNA Biotechnology Company, Esfahan, Iran) was added to the samples followed by sonication for 2 minutes. Then, chloroform was added to the samples then vortexed for 10 seconds fallowed by 5 minutes incubation at room temperature. The mixture was centrifuged at $10000 \mathrm{rpm} 8$ minutes at $4^{\circ} \mathrm{C}$ then the Transparent phase was extracted.
Afterwards, $1 \mathrm{~mL}$ from $96 \%$ ethanol was added, followed by $15-20$ minutes of incubation at $-20^{\circ}$ and 8 minutes of centrifugation at $10000 \mathrm{rpm}$ at $4^{\circ} \mathrm{C}$. After expelling the supernatant and washing the pellet with $80 \%$ ethanol, the RNA was resuspended in appropriate amount of nuclease free water. The amount of the total RNA was then measured using spectrophotometry with the absorbance at 260 nm (NanoDrop2000, Thermo Fisher Scientific Inc., Bartlesville, USA).

\section{cDNA synthesis}

First, $4 \mu \mathrm{L}$ of RNA template, $2 \mu \mathrm{L}$ of oligo(dT) 15 primer, with $1 \mu \mathrm{L}$ from deoxynucleoside triphosphate (dNTP) (RNA Biotechnology Company, Esfahan, Iran) were heated for 10 minutes at $65^{\circ} \mathrm{C}$ and then put on ice. Then, $2 \mu \mathrm{L}$ of the reaction buffer was mixed and incubated for 2 minutes at $50^{\circ} \mathrm{C}$. Afterwards, $0.5 \mu \mathrm{L}$ of $\mathrm{M}-\mathrm{MLV}$ reverse transcriptase (RNA Biotechnology Company, Esfahan, Iran) and $0.5 \mu \mathrm{L}$ of nuclease free water were added. The contents of the tube were gently mixed and incubated for 50 minutes at $50^{\circ} \mathrm{C}$ and then 15 minutes incubation at $72^{\circ} \mathrm{C}$. The cDNA was put $-20^{\circ} \mathrm{C}$ freezer till usage.

\section{PCR amplification}

To detect L. major Gp63 gene, specific primers were designed by Primer3 software. Specific primers for GAPDH gene were also used as control (Table 1). To this end, $1 \mu \mathrm{L}$ of the extracted cDNA or template DNA (for detection of the vector), $12.5 \mu \mathrm{L}$ of Master Mix (AMPLIQON, Odense, Denmark), $2 \mu \mathrm{L}$ of each primer, and nuclease free water were mixed to a final volume of $25 \mu \mathrm{L}$. The PCR program was adjusted as a 5 minutes melting step at $94^{\circ} \mathrm{C}$ and then 30 cycles of 45 seconds for denaturation at $94^{\circ} \mathrm{C}$, annealing at $54^{\circ} \mathrm{C}$, and extension at $72^{\circ} \mathrm{C}$, and a final 10 minutes extension cycle at $72^{\circ} \mathrm{C}$. PCR products were visualized using $1.5 \%$ ethidium bromide stained agarose gel.

\section{Results}

\section{Sub-cloning of L. major Gp63 gene}

The sequence of L. major Gp63 in pNUT vector was verified using sequencing and aligning with the gene sequence of gene bank. L. major Gp63 gene was then amplified with PCR by the specific primers which had two restriction sites (HindIII and EcoRI). After ligation of the gene and the pcDNA3 vector, the new vector construct was reproduced in bacteria and the vector was investigated for the presence of L. major Gp63 gene. The gene in the new construct was completely sequenced and equaled the pNUT vector. There were no mismatches between the two

Table 1. Primer pairs used for detection of GAPDH and cloning and detection of Gp63 genes

\begin{tabular}{lll}
\hline Gene & Forward primer & Reverse primer \\
\hline GAPDH & 5'-ACTCCACTCACGGCAAATTC-3' & 5'-CCTTCCACAATGCCAAAGTT-3' \\
Cloning GP63 & 5'TCTAAGCTTCACCCGATCCATGTGCCG3' & 5'AGAATTCCTGCACACTGGCGGCCGTTA'3 \\
Detecting GP63 & 5'-AACGTCTGGCAGGTCGTG-3' & 5'-AAGTGGAAGGTGACGGACAT-3' \\
\hline
\end{tabular}


sequences (Supplementary Figure).

\section{CT26 cell culture and antibiotic sensitivity assay}

CT26 colon carcinoma cells became $80 \%$ confluent within 4-5 days by refreshing the culture medium every 2-3 days (Figure 1).

To select the best dose of G418 against CT26 tumor cells, they were seeded in 24-well plates with a G418 serial concentrations . $1500 \mu \mathrm{g} / \mathrm{mL}$ was the lowest concentration of G418 which killed all the CT26 tumor cells (Figure 1).

\section{Transfection of CT26 tumor cells with L. major Gp63} CT26 tumor cells were transfected with pcDNA3 $L$. major Gp63 by Lipofectamine 2000. The Gp63 mRNA was detected with RT-PCR. The mouse GAPDH primers were used to confirm the efficacy of mRNA extraction and specific primers of L. major Gp63 were used to confirm the expression of the gene in the transfected cells (Figure 2).

\section{Establishment of stably transfected CT26 cell line}

A stably transfected CT26 cell line was established by diluting transfected cells in a 96-well plate. RT-PCR analysis of CT26 cells confirmed the expression of the $L$. major Gp63 at easily detectable levels (Figure 2).

\section{Induction of subcutaneous tumors}

To assess the potency of CT26 cells in tumor formation in vivo, the tumor cells were injected into the right flank of animals subcutaneously and the growth of the tumor mass was evaluated. In most cases, a week after the injection of CT26 cells into the right flank, the tumors were formed. When the size reached $2 \mathrm{~cm}^{2}$, the mice were euthanized and the subcutaneous tumors were carefully excised and pathology sections were prepared (Figure 3).

\section{Induction of micrometastases by intravenous injection of cancer cells}

Stable transfected CT26 cells expressing Gp63 gene were intravenously injected into the mice. The mice were divided into two control and test groups. On days 3, 7, and 14 after the injection, three mice of test and one mouse of control were sacrificed, on each day. Tissue samples were obtained from liver, lung, kidney, and colon. Total cellular RNA was extracted from the tissues and the L. major Gp63 gene was detected by RT-PCR. Gp63 gene was detected in the liver, lung, and kidney on day 3 after injection that showed the presence of injected tumor cells in these tissues but not in the colon (Figure 4). All in vivo experiments were conducted in triplicate.

\section{Discussion}

Today, the importance of micrometastasis in prognosis, treatment, and relapse of cancers has been well understood. A study on breast cancer showed a considerable reduction in the survival rate due to micrometastasis (26). A clinical study demonstrated a worse prognosis of disease in presence of bone marrow micrometastasis in in breast cancer (27). A survey on over 80000 CRC cases in the United States indicated that the elimination of at least 15 lymph nodes by colectomy prolonged the median overall survival by 54 months in node-negative patients, suggesting strong
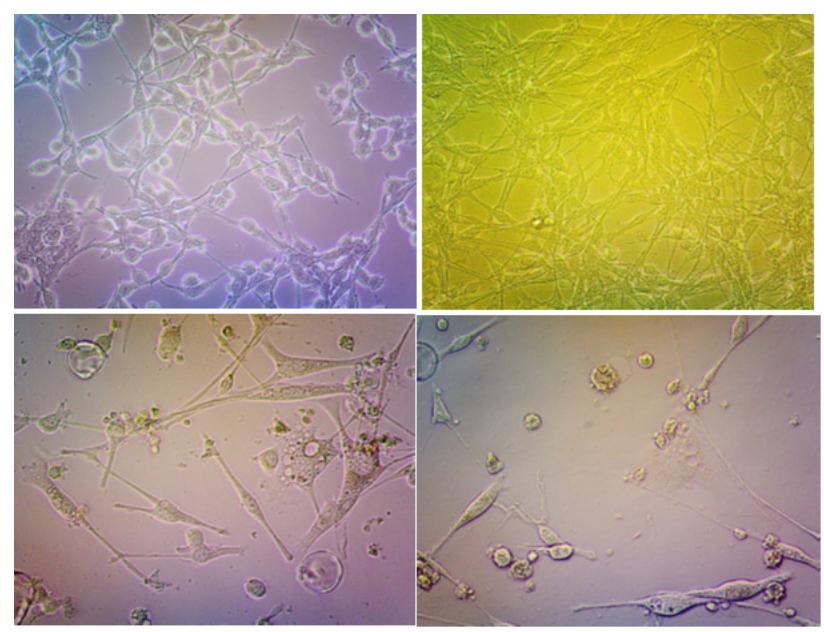

Figure 1. Antibiotic sensitivity assay for CT26 colon carcinoma cell line. CT26 tumor cells were cultured in the presence of a serial concentration of G418. Based on the results, $1500 \mu \mathrm{g} / \mathrm{mL}$ was the lowest G418 concentration at which all the CT26 tumor cells underwent apoptosis. (A) CT26 cell line at lower than $80 \%$ confluence without G418; (B) CT26 cell line at 80\% confluence without G418; (C) CT26 cell line at $80 \%$ confluence cultured in the presence of $1500 \mu \mathrm{g} / \mathrm{mL}$ G418.; (D) CT26 cell line at $80 \%$ confluence cultured in the presence of $1500 \mu \mathrm{g} / \mathrm{mL} \mathrm{G}$ G18.

\section{$\begin{array}{lllllll}1 & 2 & 3 & 4 & 5 & 6 & 7\end{array}$}

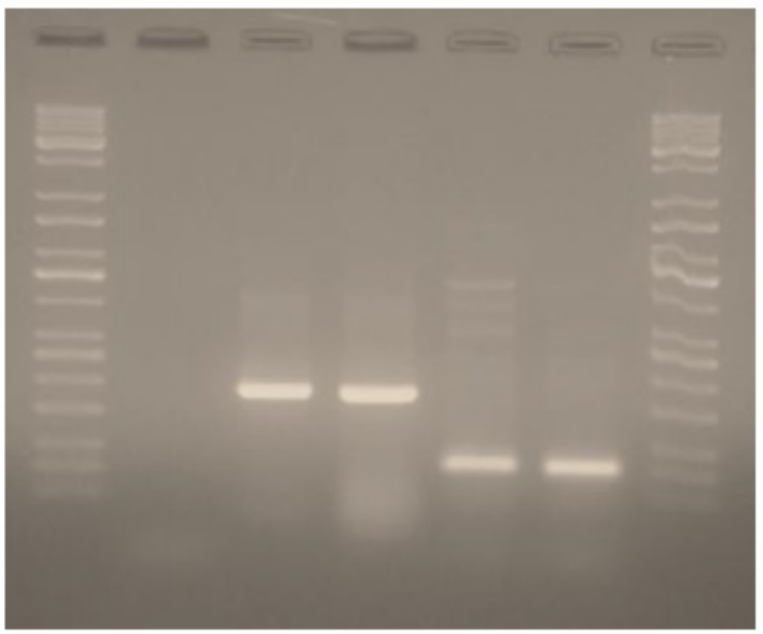

Figure 2. Confirmation of the existence of L. major Gp63 in transfected CT26 cells by RT-PCR. CT26 cells were transfected with pcDNA3 L. major Gp63 with Lipofectamine 2000 in the expression of L. major Gp63 was confirmed with RTPCR using 5'-ACATCCTCACCGACGAGAAG-3' forward and 5'-CTTGAAGTCGCCACAGATCA-3' reverse primers. The primers were expected to produce 123 bp bands. (1) standard DNA, (2) negative control, (3) GAPDH control gene, (4) GAPDH control gene, (5) L. major Gp63, (6) L. major Gp63, (7) standard DNA 
evidences of micrometastasis in lymph nodes (28). An approximate recurrence rate of $25 \%$ is estimated for cases with tumor-negative lymph nodes (28) and around 70\% of these metastatic nodes were less than $0.5 \mathrm{~cm}$ in diameter that were usually missing in pathologic dissections (29). In another study, the rate of micrometastases in the liver and lung was significantly associated with postoperative recurrence of CRCs (30).

In the present study, due to the importance of micrometastasis in different types of neoplasias and the need to establish simple and comprehensive models for extensive researches, an experimental mouse model detectable by RT-PCR was developed. It has been proven that non-detectable distant metastases are the most important causes of death in patients with colon carcinoma (31). Indeed, they are the main reasons for tumor recurrences in improved patients. Since micrometastasis is one of the most important prognostic factors for CRC (32), colon carcinoma was selected for developing the micrometastasis in the mouse model. Therefore, CT26 cell line, which is a mouse colon carcinoma, was used as a cell line-based allograft. Allograft models in mice are raised from the inoculation of tumor cells derived from mice. Unlike xenograft models in that human tumor cells are transplanted to immune-compromised or immunedeficient mice, allograft models leave the immune system intact and do not need compromising the host immune system to prevent rejection of the tumor, thus showing more similarity to the cancer environment. Therefore, using a syngeneic mouse model for studying the tumor

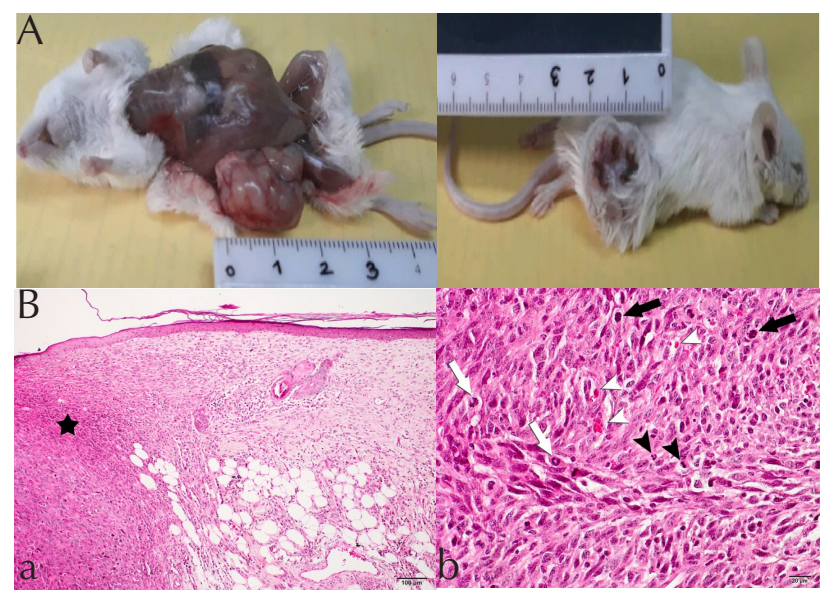

Figure 3. Induction of CT26 tumor in BALB/c mice. $10^{6} \mathrm{CT} 26$ cells were injected into the right flank of 6 -8-week BALB/C mice subcutaneously. (A) tumor mass after 4 weeks, (B) tumor section for pathological assessment; (a) The micrograph shows a welldeveloped neoplastic mass in the dermis surrounded by infiltrated inflammatory cells (asterisk). (b) Malignant nature of the tumor is associated with distinct histomorphologic changes e.g., pleomorphism, hyperchromasia (black arrows), abundant mitotic figures (white arrows), apoptotic bodies (black arrowheads), and angiogenesis (white arrowheads).

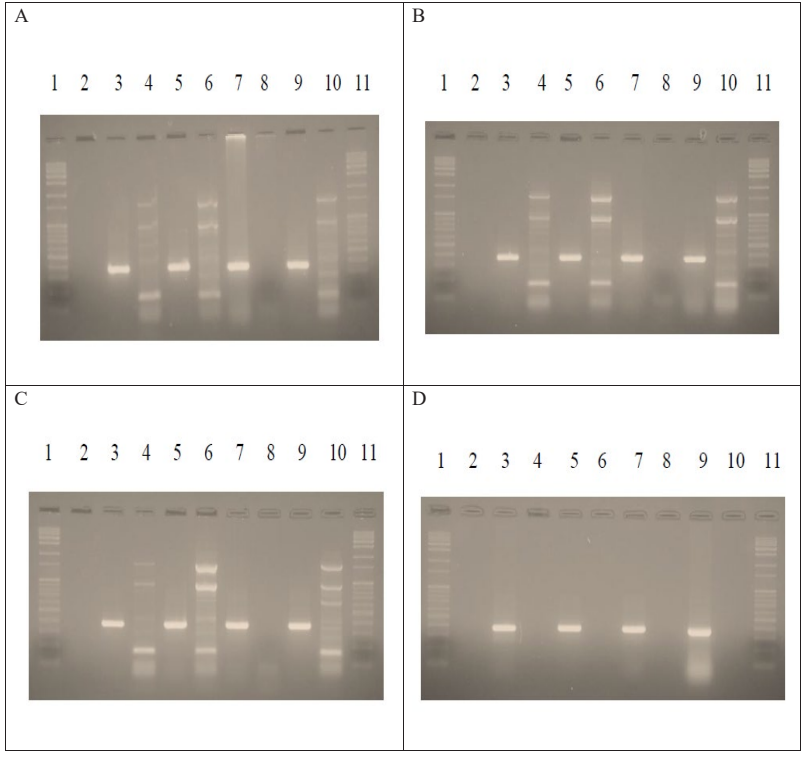

Figure 4. Transfection of CT26 tumor cells with L. major Gp63 in BALB/c mice. $1 \times 10^{6}$ stably transfected CT26/L. major Gp63 cells were intravenously injected into BALB/C mice and the expression of Gp63 was detected using RT-PCR in different organs targeted for metastasis of the tumor cells on days 3, 7, and 14 .

(A) Samples taken on day 3: 1 and 11-Standard DNA, 2- Negative control, 3- Liver GAPDH, 4- Liver Gp63, 5- Lung GAPDH, 6- Lung Gp63, 7- Colon GAPDH, 8- Colon Gp63, 9- Kidney GAPDH, 10Kidney Gp63. (B) Samples taken on day 7: 1 and 11- Standard DNA, 2- Negative control, 3- Liver GAPDH, 4- Liver Gp63, 5Lung GAPDH, 6- Lung Gp63, 7- Colon GAPDH, 8- Colon Gp63, 9- Kidney GAPDH, 10- Kidney Gp63. (C) Samples taken on day 14: 1 and 11-Standard DNA, 2- Negative control, 3- Liver GAPDH, 4- Liver Gp63, 5- Lung GAPDH, 6- Lung Gp63, 7- Colon GAPDH, 8- Colon Gp63, 9- Kidney GAPDH, 10- Kidney Gp63. (D) Samples from mice intravenously injected with $100 \mu \mathrm{L}$ of PBS. 1 and 11 Standard DNA, 2- Negative control, 3- Liver GAPDH, 4- Liver Gp63, 5- Lung GAPDH, 6- Lung Gp63, 7-Colon GAPDH, 8- Colon Gp63, 9- Kidney GAPDH, 10- Kidney Gp63.

metastasis behavior clearly shows many features of the early tumor progress and also the metastatic outspread without interfering with the host's adaptive immune system (33).

Generally, to study metastasis, spontaneous and experimental mouse models are used. In spontaneous metastasis models, a primary tumor is formed, and then the metastatic cells spread to peripheral sites spontaneously. This model is capable of tracking the tumor cells in the animals. The main advantage of this model is that they permit the whole metastatic process to be modeled but poor clinical settings for metastasis restrict its usage. In addition, due to the long latency and the need for the primary tumor to be removed for metastasis development, only limited numbers of cell lines such as mammary tumors and melanomas can be studied by spontaneous models. Experimental models for metastasis are also practical to assess the metastatic potency of tumor cells in a particular organ following intravascular injection. These models are often practical to survey late stages of the micrometastatic process and for better definition of organs targeted for 
homing of metastatic cells not the entire metastatic cascade. In this model, the cells can be injected into different sites such as intracardiac, intraportal, intracarotid and lateral tail veins in the mouse. Because of the lower fatality rate and no need for anesthesia, the injection of tumor cells into the tail vein is one of the easiest approaches used in tumor metastatic models. In addition, this injection approach is rapid, low-cost, and applicable to a wide range of tumor cells. The injection of tumor cells to the tail vein causes lung metastases minutes after the injection (33). Most of the tumor cells retain in lungs but a few cells pass through the lungs and spread to some other organs (16). This metastatic behavior of tumor cells was also confirmed in the model presented in this study where the metastatic cells were detected in the liver, kidneys, and lungs 3, 7, and 14 days after tumor cell injection but not observed in colon. This model can be used in almost all types of neoplasias and different organs can be studied as micrometastatic targets.

In a study on targeted therapy of colonic cancer micrometastases, the tumors were initiated by injecting the cell suspension into the caudal tail vein and survival curves were obtained for tumor bearing animals subjected to targeted therapy 1,7 , and, 14 days after tumor cell implantation. The micrometastatic nodules were observed on days 7 and 14 in the lungs (34) which are consistent with our observations. However, in another study, a micrometastatic lung model was created by injecting the mice with a suspension of GW-39 tumor cells in the tail vein and the micrometastasis was detected after 14 days (23). Wetterwald et al compared intravenous and intracardiac injections of a human breast carcinoma cell line (MDAMB-231) for inducing metastases with bioluminescent reporter imaging. They demonstrated that despite the high rate of fatality and signs of distress in intracardiac injection, all of the survived mice developed bone metastases. In contrast, none of the intravenously injected mice showed soft tissue and bone metastases within the total trial time (4 weeks) (12). Likewise, Peyruchaud et al established a sub-clone of MDA-MB-231 cells by injecting tumor cells expressing green fluorescent protein into the tail vein of mice. They reported the growth of these cells implanted in the bone environment (35). In another study, an experimental liver metastasis model was developed by sterile surgery on the left flank of the mouse (36). In comparison with the injection into the tail vein, this model was cumbersome and time-consuming. Luzzi et al designed a mouse model for liver metastasis using in vivo video microscopy and immunohistochemical markers for proliferation. They showed that by day 3, some micrometastases consisting of 4 to 16 cells were developed (10). These data confirm our observations on the formation of micrometastasis on day 3 after the injection.

In recent studies, different tumor markers have been used for detection of tumor micrometastasis. In a study, carcinoembryonic antigen was detected by RT-PCR in the lymph nodes of gastrointestinal or breast carcinoma patients. All normal mucosal tissues as well as carcinomas produced carcinoembryonic antigen mRNA but no amplification was seen in none of control lymph nodes and only 30 out of 117 lymph nodes showed histological signs of carcinoma cells (20). A study in Germany checked the specificity of cytokeratin 20 (CK20) mRNA for tracing circulating tumor cells in peripheral blood and bone marrow of patients. CK20 expressing cells were recognized in granulocytes which expressed the gene on a background level. Therefore, the expression of the gene in normal granulocytes should be considered to distinguish the normal cells from the circulating micrometastases (37). Therefore, more specific markers with no expression in normal tissues are needed for better diagnosis of micrometastases. Therefore, in the present study, to generate a potent marker for tracing micrometastatic cells in tissues, CT26 cells were transfected with L. major Gp63 cDNA. Leishmania Gp63 gene was cloned and expressed in cells to induce cell-mediated immune response to Leishmania species in different vaccine studies (38). In addition, CT26 cells transfected with $L$. major Gp63 were already applied to rate the cytotoxicity in a standard 4-hour 51Cr-release cytotoxicity assay (39). Although transfection of Gp63 gene has already been used for prevention and therapeutic goals against leishmaniasis in different studies, this is the first report on the use of $L$. major Gp63 as a marker for cancer cells for follow-up and detection of micrometastasis with RT-PCR technique in a mouse model.

RT-PCR has the potential to detect micrometastases at extremely low frequencies. In a study, this technique has been used to detect a pancreatic cancer marker in peritoneal washings as an indicator of the presence of peritoneal micrometastasis. The researchers demonstrated that this method was comparable to cytology results (19). It seems that RT-PCR can be one of the best techniques to detect cancer cells in tissues due to the low number of tumor cells in micrometastasis. To detect tumor micrometastasis, the tumor cells were transfected with $G p 63$ cDNA as a foreign gene which exists neither in cancer cells nor in the mouse tissue. To improve the efficacy of RT-PCR in detecting Gp63 gene as a marker of CT26 cells, the gene was inserted into pcDNA3 vector which is a potent mammalian expression vector with a high copy number and contain cytomegalovirus promoter which induces an excessive level of gene expression.

\section{Conclusion}

CT26 tumor cells stably expressing Gp63 are a powerful in vivo tool to investigate micrometastasis behaviors in cancer research. Although in vitro studies are necessary, only in vivo models can evaluate the potency and efficiency of cancer therapy in the complex environment of living animals. In this study, we introduced a micrometastatic mouse model using CT26 tumor cells stably transfected with L. major 
Gp63 that can be detected by RT-PCR as a reliable model to study micrometastasis of cancers to contribute to current and future cancer research.

Conflict of interests

The authors declare no conflict of interests.

Ethical Approval

All experiments were carried out according to Ethical Guidelines for the Care and Use of Laboratory Animals. This study was approved by the Ethics Committee of Bu-Ali Sina University (IR.UMSHA. REC.1395,120).

Acknowledgments

We thank Miss S. Azami and Ms. S. Hamoon Navard for their scientific assistance in preparing cell culture and working with animals, respectively. We also thank the Research Deputy of Bu-Ali Sina University for financially supporting the study (grant number 97-4 dated 97/7/15).

\section{Supplementary Materials}

Supplementary file contains Alignment of L. major Gp63 Sequence Cloned in $p C D N A 3$ and $p N U T$ Vectors.

\section{References}

1. Favoriti P, Carbone G, Greco M, Pirozzi F, Pirozzi RE, Corcione F. Worldwide burden of colorectal cancer: a review. Updates Surg. 2016;68(1):7-11. doi: 10.1007/s13304-016-0359-y.

2. He C, Duan X, Guo N, Chan C, Poon C, Weichselbaum RR, et al. Core-shell nanoscale coordination polymers combine chemotherapy and photodynamic therapy to potentiate checkpoint blockade cancer immunotherapy. Nat Commun. 2016;7:12499. doi: 10.1038/ncomms12499.

3. Ansari R, Mahdavinia M, Sadjadi A, Nouraie M, Kamangar $F$, Bishehsari F, et al. Incidence and age distribution of colorectal cancer in Iran: results of a population-based cancer registry. Cancer Lett. 2006;240(1):143-7. doi: 10.1016/j. canlet.2005.09.004.

4. Jemal A, Bray F, Center MM, Ferlay J, Ward E, Forman D. Global cancer statistics. CA Cancer J Clin. 2011;61(2):69-90. doi: 10.3322/caac.20107.

5. Murphy N, Ward HA, Jenab M, Rothwell JA, Boutron-Ruault MC, Carbonnel F, et al. Heterogeneity of colorectal cancer risk factors by anatomical subsite in 10 European countries: a multinational cohort study. Clin Gastroenterol Hepatol. 2019;17(7):1323-31.e6. doi: 10.1016/j.cgh.2018.07.030.

6. Fakheri $\mathrm{H}$, Janbabai G, Bari Z, Eshqi F. The epidemiologic and clinical-pathologic characteristics of colorectal cancers from 1999 to 2007 in Sari, Iran. Journal of Mazandaran University of Medical Sciences. 2008;18(67):58-66. [Persian].

7. Russo AG, Andreano A, Sartore-Bianchi A, Mauri G, Decarli A, Siena S. Increased incidence of colon cancer among individuals younger than 50 years: a 17 years analysis from the cancer registry of the municipality of Milan, Italy. Cancer Epidemiol. 2019;60:134-40. doi: 10.1016/j.canep.2019.03.015.

8. Woelfle U, Cloos J, Sauter G, Riethdorf L, Jänicke F, van Diest $\mathrm{P}$, et al. Molecular signature associated with bone marrow micrometastasis in human breast cancer. Cancer Res. 2003;63(18):5679-84.

9. Fiegle E, Doleschel D, Koletnik S, Rix A, Weiskirchen R, Borkham-Kamphorst E, et al. Dual CTLA-4 and PD-L1 blockade inhibits tumor growth and liver metastasis in a highly aggressive Orthotopic mouse model of Colon Cancer. Neoplasia. 2019;21(9):932-44. doi: 10.1016/j.neo.2019.07.006.
10. Luzzi KJ, MacDonald IC, Schmidt EE, Kerkvliet N, Morris VL, Chambers AF, et al. Multistep nature of metastatic inefficiency: dormancy of solitary cells after successful extravasation and limited survival of early micrometastases. Am J Pathol. 1998;153(3):865-73. doi: 10.1016/s0002-9440(10)65628-3.

11. Zhou Y, Zhang GJ, Wang J, Zheng KY, Fu W. Current status of lymph node micrometastasis in gastric cancer. Oncotarget. 2017;8(31):51963-9. doi: 10.18632/oncotarget.17495.

12. Wetterwald A, van der Pluijm G, Que I, Sijmons B, Buijs J, Karperien $M$, et al. Optical imaging of cancer metastasis to bone marrow: a mouse model of minimal residual disease. Am J Pathol. 2002;160(3):1143-53. doi: 10.1016/s00029440(10)64934-6.

13. Hum NR, Martin KA, Malfatti MA, Haack K, Buchholz BA, Loots GG. Tracking tumor colonization in xenograft mouse models using accelerator mass spectrometry. Sci Rep. 2018;8(1):15013. doi: 10.1038/s41598-018-33368-0.

14. Hoffman R. Green fluorescent protein imaging of tumour growth, metastasis, and angiogenesis in mouse models. Lancet Oncol. 2002;3(9):546-56. doi: 10.1016/s14702045(02)00848-3.

15. Bilchik AJ, Hoon DS, Saha S, Turner RR, Wiese D, DiNome $M$, et al. Prognostic impact of micrometastases in colon cancer: interim results of a prospective multicenter trial. Ann Surg. 2007;246(4):568-75; discussion 75-7. doi: 10.1097/ SLA.0b013e318155a9c7.

16. MacDonald IC, Groom AC, Chambers AF. Cancer spread and micrometastasis development: quantitative approaches for in vivo models. Bioessays. 2002;24(10):885-93. doi: 10.1002/ bies.10156.

17. Isnard A, Shio MT, Olivier M. Impact of Leishmania metalloprotease GP63 on macrophage signaling. Front Cell Infect Microbiol. 2012;2:72. doi: 10.3389/fcimb.2012.00072.

18. Dalby B, Cates S, Harris A, Ohki EC, Tilkins ML, Price PJ, et al. Advanced transfection with Lipofectamine 2000 reagent: primary neurons, siRNA, and high-throughput applications. Methods. 2004;33(2):95-103. doi: 10.1016/j. ymeth.2003.11.023.

19. Sato K, Mori R, Hiroshima Y, Oba MS, Matsuyama R, Bouvet $M$, et al. RT-PCR of peritoneal washings predicts peritoneal pancreatic cancer recurrence. J Surg Res. 2018;226:122-30. doi: 10.1016/j.jss.2017.11.009.

20. Mori M, Mimori K, Inoue H, Barnard GF, Tsuji K, Nanbara S, et al. Detection of cancer micrometastases in lymph nodes by reverse transcriptase-polymerase chain reaction. Cancer Res. 1995;55(15):3417-20.

21. Xiang R, Lode HN, Dolman CS, Dreier T, Varki NM, Qian $X$, et al. Elimination of established murine colon carcinoma metastases by antibody-interleukin 2 fusion protein therapy. Cancer Res. 1997;57(21):4948-55.

22. Guba M, von Breitenbuch P, Steinbauer M, Koehl G, Flegel S, Hornung $M$, et al. Rapamycin inhibits primary and metastatic tumor growth by antiangiogenesis: involvement of vascular endothelial growth factor. Nat Med. 2002;8(2):128-35. doi: 10.1038/nm0202-128.

23. Sharkey RM, Blumenthal RD, Behr TM, Wong GY, Haywood $L$, Forman D, et al. Selection of radioimmunoconjugates for the therapy of well-established or micrometastatic colon carcinoma. Int J Cancer. 1997;72(3):477-85. doi: 10.1002/ (sici)1097-0215(19970729)72:3<477::aid-ijc16>3.0.co;2-9.

24. Zeelenberg IS, Ruuls-Van Stalle L, Roos E. The chemokine receptor CXCR4 is required for outgrowth of colon carcinoma micrometastases. Cancer Res. 2003;63(13):3833-9.

25. Rezvan H. Molecular cloning of Leishmania major gp63 Gene in BALB/C Mouse CT26 Cell Line. Zahedan Journal of Research 
in Medical Sciences. 2015;17(5):1-5. doi: 10.17795/zjrms958.

26. Truong PT, Vinh-Hung V, Cserni G, Woodward WA, Tai P, Vlastos

G. The number of positive nodes and the ratio of positive to excised nodes are significant predictors of survival in women with micrometastatic node-positive breast cancer. Eur J Cancer. 2008;44(12):1670-7. doi: 10.1016/j.ejca.2008.05.011.

27. Braun S, Vogl FD, Naume B, Janni W, Osborne MP, Coombes $\mathrm{RC}$, et al. A pooled analysis of bone marrow micrometastasis in breast cancer. N Engl J Med. 2005;353(8):793-802. doi: 10.1056/NEJMoa050434.

28. Chen SL, Bilchik AJ. More extensive nodal dissection improves survival for stages I to III of colon cancer: a population-based study. Ann Surg. 2006;244(4):602-10. doi: 10.1097/01. sla.0000237655.11717.50.

29. Rodriguez-Bigas MA, Maamoun S, Weber TK, Penetrante RB, Blumenson LE, Petrelli NJ. Clinical significance of colorectal cancer: metastases in lymph nodes $<5 \mathrm{~mm}$ in size. Ann Surg Oncol. 1996;3(2):124-30. doi: 10.1007/bf02305790.

30. Yang KM, Park IJ, Lee JL, Kim CW, Yoon YS, Lim SB, et al. Benefits of repeated resections for liver and lung metastases from colorectal cancer. Asian J Surg. 2020;43(1):102-9. doi: 10.1016/j.asjsur.2019.03.002.

31. Viehl CT, Weixler B, Guller U, Dell-Kuster S, Rosenthal R, Ramser M, et al. Presence of bone marrow micro-metastases in stage I-III colon cancer patients is associated with worse disease-free and overall survival. Cancer Med. 2017;6(5):91827. doi: 10.1002/cam4.1056.

32. Jang KU, Kim CW, Kim KH, Lim SB, Yu CS, Kim TW, et al. Prognostic factors in terms of the number of metastatic nodules in patients with colorectal cancer liver metastases. Ann Coloproctol. 2016;32(3):92-100. doi: 10.3393/ ac.2016.32.3.92.

33. Gómez-Cuadrado L, Tracey N, Ma R, Qian B, Brunton VG. Mouse models of metastasis: progress and prospects. Dis Model Mech. 2017;10(9):1061-74. doi: 10.1242/dmm.030403.

34. Blumenthal RD, Sharkey RM, Haywood L, Natale AM, Wong GY, Siegel JA, et al. Targeted therapy of athymic mice bearing GW39 human colonic cancer micrometastases with 1311-labeled monoclonal antibodies. Cancer Res. 1992;52(21):6036-44.

35. Peyruchaud O, Winding B, Pécheur I, Serre CM, Delmas $P$, Clézardin P. Early detection of bone metastases in a murine model using fluorescent human breast cancer cells: application to the use of the bisphosphonate zoledronic acid in the treatment of osteolytic lesions. J Bone Miner Res. 2001;16(11):2027-34. doi: 10.1359/jbmr.2001.16.11.2027.

36. Wilmanns C, Fan D, O'Brian CA, Bucana CD, Fidler IJ. Orthotopic and ectopic organ environments differentially influence the sensitivity of murine colon carcinoma cells to doxorubicin and 5-fluorouracil. Int J Cancer. 1992;52(1):98104. doi: 10.1002/ijc.2910520118.

37. Jung R, Petersen K, Krüger W, Wolf M, Wagener C, Zander A, et al. Detection of micrometastasis by cytokeratin 20 RT-PCR is limited due to stable background transcription in granulocytes. Br J Cancer. 1999;81(5):870-3. doi: 10.1038/sj.bjc.6690778.

38. Sinha S, Sundaram S, Singh AP, Tripathi A. A gp63 based vaccine candidate against visceral leishmaniasis. Bioinformation. 2011;5(8):320-5. doi: 10.6026/97320630005320.

39. Ali SA, Rezvan H, McArdle SE, Khodadadi A, Asteal FA, Rees RC. CTL responses to Leishmania mexicana gp63-cDNA vaccine in a murine model. Parasite Immunol. 2009;31(7):37383. doi: 10.1111/j.1365-3024.2009.01111.x. 